\title{
Strong interference between decay channels and valence-electron rearrangements in core-hole spectroscopy
}

\author{
J. Zaanen and G. A. Sawatzky \\ Laboratory for Physical Chemistry, University of Groningen, Nijenborgh 16, NL-9747 AG Groningen, The Netherlands
}

(Received 14 August 1985)

\begin{abstract}
In the analysis of core-hole spectra it is usually assumed that the response of the valence electrons upon the creation of a core hole can be treated independent of the subsequent decay of the core hole. We show that for near-threshold decay processes this is no longer true and that the modification of the satellite spectra is considerable even for relative slow decay processes. Taking the $2 p \mathrm{x}$-ray photoemission spectra of $\mathrm{NiBr}_{2}$ as an example, we show that the large difference between the $2 p_{3 / 2}$ and $2 p_{1 / 2}$ spectra of transition-metal compounds is due to the near-threshold Coster-Kronig decay of the $2 p_{1 / 2}$ hole.
\end{abstract}

\section{INTRODUCTION}

Recently, several theoretical models have been proposed to interpret core-hole line shapes and, in particular, satellite structures, in terms of the valence-electronic structure of materials. This is an extremely important development since if applicable to real materials we can obtain quite detailed information about the valence-electronic structure from core-line spectroscopies. Examples for which the theoretical models have had considerable success are the $\mathrm{X}$-ray photoemission spectroscopy (XPS) spectra of copper dihalides, ${ }^{1,2}$ cerium compounds, ${ }^{3,4}$ scandium and titanium halides, ${ }^{5}$ and $\mathrm{La}$ and Ce intermetallic compounds. ${ }^{6,7}$

These theoretical models treat in various approximations the valence-band structure and the valencevalence-electron-electron interactions, as well as the core-hole-valence-electron Coulomb interactions. The approximations made in the Hamiltonians used to describe the above are usually well founded for the particular systems studied. There is, however, one commonly used approximation which has received little theoretical attention and which can lead to incorrectly calculated line shapes and can even lead to large errors in the various parameters appearing in the models when comparison is made to experimental results. The approximation we refer to is the neglect of the details of the core-hole decay processes. In the above models the decay processes are usually included merely by convoluting the theoretical spectra with a Lorentzian.

In our recent confrontation with the interpretation of $2 p$ XPS satellite structures in the transition-metal dihalides, we tried to understand the large difference in the $2 p_{3 / 2}$ and $2 p_{1 / 2}$ satellite structure. This problem, reproduced in Fig. 1 for the Ni dihalides, is especially evident in all the $\mathrm{Ni}, \mathrm{Co}, \mathrm{Fe}$, and $\mathrm{Mn}$ compounds. ${ }^{8-18}$ The first obvious possible explanation is the influence of final-state multiplet splittings, but we found that this could certainly not explain the results. ${ }^{19-23}$

The purpose of this paper is to show that under certain conditions, as met in the above examples due to the Coster-Kronig decay of the $2 p_{1 / 2}$ hole, the relaxation of the valence electrons and the decay of the core-hole can- not be treated independently. The interference between both processes can even be so strong that the use of intensities and line positions in terms of theories which do not explicitly include the decay continua can lead to quite incorrect values for relevant parameters.

We will show that the decay process will affect the overall satellite line shape if the decay process is near threshold, even though the lifetime width is only 10-20\% of the line splittings. By near threshold we mean that the Auger electron is emitted at low kinetic energy. From the work of Ohno and Wendin (for a review, see Ref. 24), it is well known that fast decay processes can effect tremendously the shape of single-component XPS core lines as is the case for $4 s$ and $4 p$ lines of the elements surrounding $\mathrm{Xe}$ which decay by giant Coster-Kronig channels. However, the problem of multicomponent spectra is considerably more complicated because the full screening dynamics before and after the decay has to be considered in order to give a proper description of the XPS line shape. For this reason this paper can be considered a generalization for near-threshold processes of a

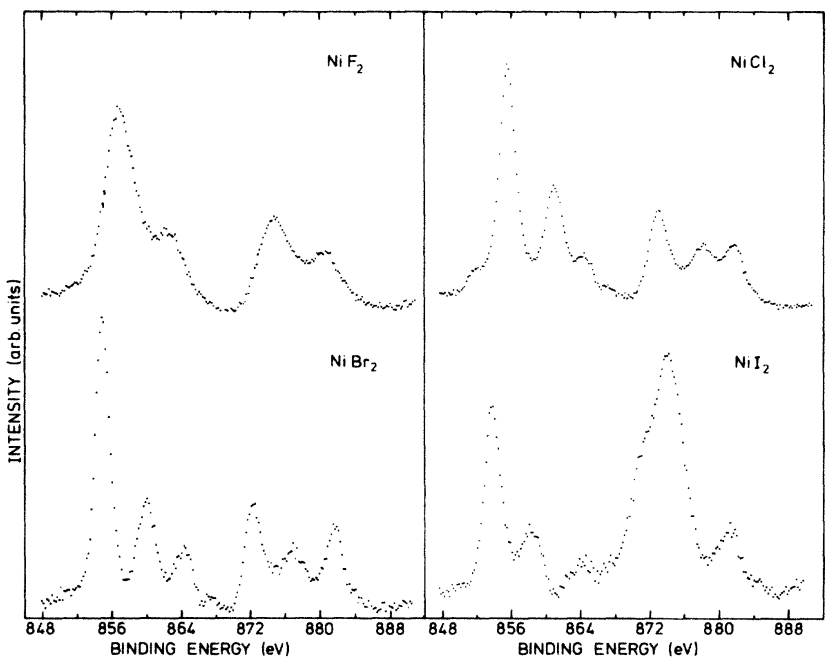

FIG. 1. Ni $2 p$ XPS spectra of the Ni dihalides. In $\mathrm{NiI}_{2}$ the $2 p_{3 / 2}$ region is eclipsed by the $\mathrm{I} 3 p_{3 / 2}$ line. 
part of the dynamical theory of the Auger process as developed by Gunnarsson and Schönhammer. ${ }^{25}$

The organization of this paper is as follows: In Sec. II we go into the problem of spectral line shapes of systems containing a number of mutually coupled discrete states which, in turn, are coupled to many continua. Although the formal aspects of the theory are not new, ${ }^{26,27}$ this section serves to give a physical intuition, using simple models, about the kind of effects which can be expected.

In Sec. III the theory is applied to the situation encountered for the transition-metal and rare-earth compounds. Within the limitations of the cluster approach, ${ }^{1-3,28}$ we illuminate here the basic physics involved and the conditions under which these interference effects will be important. Finally, in Sec. IV the theory of the preceding section is applied to the $2 p$ XPS spectra of $\mathrm{NiBr}_{2}$. In this section we show that we have, in principle, enough knowledge about the large-energy-scale valence-electronic structure in the presence of a core hole of this compound to determine the thresholds of the Coster-Kronig decay continua. Indeed, it appears that the Coster-Kronig process meets the above-mentioned criteria and explains the strong difference in the $2 p_{3 / 2}$ and $2 p_{1 / 2}$ satellite structure.

\section{THEORY OF SPECTROSCOPIC LINE SHAPES}

For spectroscopies, in general, we must consider transitions from a ground state to a set of final states. The final states which can be reached can, by a suitable choice of basis functions, be divided into discrete states and continuum states. The Hamiltonian can be factorized as follows:

$$
\begin{aligned}
H= & \sum_{i}|i\rangle E_{i}\left\langle i\left|+\sum_{i, j}\right| i\right\rangle T_{i j}\left\langle j\left|+\sum_{k_{n}}\right| k_{n}\right\rangle E_{k_{n}}\left\langle k_{n}\right| \\
& +\sum_{i k_{n}}|i\rangle V_{i k_{n}}\left\langle k_{n}\right|,
\end{aligned}
$$

where $E_{i}$ are the energies of the discrete states, $E_{k_{n}}$ the energies of the various possible continuum states, and $T_{i j}$ describes a coupling of the discrete states and $V_{\boldsymbol{k}_{n} i}$ describes the coupling of discrete states to the various continua.

The spectral distribution of transitions to the states described by $H$ will be determined by

$$
\begin{gathered}
I(\omega)=\frac{1}{\pi} \operatorname{Im}\left(\sum_{i, j} A_{i}^{*} A_{j} G_{i j}+\sum_{i, k_{n}} A_{i}^{*} A_{k_{n}} G_{i k_{n}}\right. \\
\left.+\sum_{k_{n}, k_{n^{\prime}}^{\prime}} A_{k_{n}}^{*} A_{k_{n^{\prime}}^{\prime}} G_{k_{n} k_{n^{\prime}}^{\prime}}\right),
\end{gathered}
$$

where

$$
G_{i j}=\left\langle i\left|\frac{1}{z-H}\right| j\right\rangle,
$$

with $z=\omega-i \delta$ and $A$ are the transition matrix elements from the ground state which depend on the spectroscopy. The second term in (2) is largely responsible for the wellknown Fano line shape ${ }^{27}$ for situations in which both the discrete and continuum state are spectroscopically accessible and are in the same energy region.

Here we want to consider the case in which only the discrete states are spectroscopically accessible but where these states couple to one or more continua, describing the decay processes. In this case we are only interested in $G_{i j}$ with $i$ and $j$ referring to the discrete states. The continua then refer to decaylike channels for the discrete states.

Using the Dyson equation, we can write

$$
\begin{aligned}
& G_{i j}=G_{i i}^{0} \delta_{i j}+G_{i i}^{0} \sum_{p} T_{i p} G_{p j}+G_{i i}^{0} \sum_{k_{n}} V_{i k_{n}} G_{k_{n} j}, \\
& G_{k_{n} j}=G_{k_{n} k_{n}}^{0} \sum_{l} V_{k_{n} l} G_{l j},
\end{aligned}
$$

or

$G_{i j}=G_{i j}^{0} \delta_{i j}+G_{i i}^{0} \sum_{l}\left(T_{i l}+\sum_{n, k} V_{i k_{n}} G_{k_{n} k_{n}}^{0} V_{k_{n} l}\right) G_{l, j}$,

where

$$
G_{k_{n} k_{n}}^{0}=\sum_{k_{n}} \frac{1}{\omega-\varepsilon_{k_{n}}}
$$

and

$$
G_{i i}^{0}=\frac{1}{\omega-\varepsilon_{i}} .
$$

Equation (5) describes the spectral distribution of the discrete states having projected out the continuum states. We note that Eq. (5) can be described by considering only the discrete states with an effective Hamiltonian

$H=\sum_{i}|i\rangle \varepsilon_{i}^{\prime}\langle i|+\sum_{i j}\left(|i\rangle T_{i j}^{\prime}\langle j|+| j\rangle T_{j i}^{\prime}\langle i|\right)$,

where

$$
\begin{aligned}
& \varepsilon_{i}^{\prime}=\varepsilon_{i}+\operatorname{Re}\left(\sum_{k_{\omega}, n} \frac{\left|v_{k_{i} n}\right|^{2}}{\omega-\varepsilon_{k_{n}}}\right)+i \Gamma_{i}, \\
& T_{i j}^{\prime}=T_{i j}+\operatorname{Re}\left(\sum_{k_{n}, n} \frac{V_{i k_{n}} V_{k_{n} j}}{\omega-\varepsilon_{k_{n}}}\right)+i \Gamma_{i j}, \\
& \Gamma_{i}=\operatorname{Im}\left(\sum_{k_{n}, n} \frac{\left|V_{k_{n} i}\right|^{2}}{\omega-\varepsilon_{k_{n}}}\right), \\
& \Gamma_{i j}=\operatorname{Im}\left[\sum_{k_{n}, n} \frac{V_{i k_{n}} V_{k_{n} j}}{\omega-\varepsilon_{k_{n}}}\right) .
\end{aligned}
$$

We note first that $\varepsilon_{i}^{\prime}$ and $T_{i j}^{\prime}$ contain an imaginary part and also that $T_{i j}^{\prime}=T_{j i}^{\prime}$ for $V_{k_{n} i}$ real and not, as usually is the case, $T_{i j}^{\prime}=\left(T_{j i}^{\prime}\right)^{*}$. Note also that the $T_{i j}^{\prime}$ are real unless the discrete states couple to the same continuum.

In the usual treatment of XPS the coupling to the decay continua is replaced by a single decay rate so that it is assumed that $\Gamma_{i}=\Gamma_{j}$ and $\Gamma_{i j}=0$. We will refer to this as the optical potential approximation.

Since it is well known that the relative intensities of 
spectral components depend strongly on the matrix elements mixing the discrete states, we can expect large deviations from the optical potential model if $T_{i j}^{\prime} \neq T_{i j}$. This will occur if the discrete states can couple to the same continuum. Also, as we will show below, deviations from the optical potential model can be expected if $\Gamma_{i} \neq \Gamma_{j}$.

To illustrate the deviations which can occur from the optical potential model, we resort to a simple example of two discrete states coupled to a number of continua. We will here also assume that the continuum densities of states are constant. We then have the relations

$$
\begin{aligned}
& G_{11}=\left(\omega-\varepsilon_{2}-i \Gamma_{2}\right) \phi, \\
& G_{22}=\left(\omega-\varepsilon_{1}-i \Gamma_{1}\right) \phi, \\
& G_{12}=G_{21}=\left(T+i \Gamma_{12}\right) \phi,
\end{aligned}
$$

with

$$
\phi=\frac{1}{\left(\omega-\varepsilon_{1}-i \Gamma_{1}\right)\left(\omega-\varepsilon_{2}-i \Gamma_{2}\right)-\left(T+i \Gamma_{12}\right)^{2}} .
$$

In order to see physically what happens, we define a transformation

$$
U=\left(\begin{array}{cc}
\cos \theta & \sin \theta \\
-\sin \theta & \cos \theta
\end{array}\right)
$$

so as to diagonalize $G$.

Defining the diagonal components of $G$ as $G_{a a}$ and $G_{b b}$, we find that $G_{a b}=0$ for

$$
\begin{aligned}
(\sin \theta \cos \theta)\left[\varepsilon_{2}-\varepsilon_{1}+i\left(\Gamma_{2}\right.\right. & \left.\left.-\Gamma_{1}\right)\right] \\
& +\left(\cos ^{2} \theta-\sin ^{2} \theta\right)\left(T+i \Gamma_{12}\right)=0,
\end{aligned}
$$

or

$$
\tan (2 \theta)=-\frac{2\left(T+i \Gamma_{12}\right)}{\varepsilon_{2}-\varepsilon_{1}+i \Delta \Gamma},
$$

where

$$
\Delta \Gamma=\Gamma_{2}-\Gamma_{1}
$$

This looks similar to the solution in the absence of decay for a two-level problem, as, for example, treated by van der Laan et al., ${ }^{1}$ except that now the angle $\theta$ has become complex. We see immediately from Eq. (14) that for $\Gamma_{12}=\Delta \Gamma=0$ one can use the optical potential model to describe the problem.

In terms of our original basis set, the spectrum will be given by Eq. (2). With

$$
\begin{aligned}
& G_{11}=\left(\sin ^{2} \theta\right) G_{a a}+\left(\cos ^{2} \theta\right) G_{b b}, \\
& G_{22}=\left(\cos ^{2} \theta\right) G_{a a}+\left(\sin ^{2} \theta\right) G_{b b}, \\
& G_{12}=(\sin \theta \cos \theta)\left(G_{b b}-G_{a a}\right),
\end{aligned}
$$

or defining $\theta=\chi+i \phi$,

$$
\begin{aligned}
G_{11}= & \frac{1}{2} G_{a a}[1+\cos (2 \chi) \cosh (2 \phi)] \\
& +\frac{1}{2} G_{b b}[1-\cos (2 \chi) \cosh (2 \phi)] \\
& +\frac{i}{2} \sin (2 \chi) \sinh (2 \phi)\left(G_{b b}-G_{a a}\right), \\
G_{12}= & \frac{1}{2}[\sin (2 \chi) \cosh (2 \phi) \\
& \quad+i \cos (2 \chi) \sinh (2 \phi)]\left(G_{b b}-G_{a a}\right), \\
G_{22}= & \frac{1}{2} G_{a a}[1-\cos (2 \chi) \cosh (2 \phi)] \\
& +\frac{1}{2} G_{b b}[1+\cos (2 \chi) \cosh (2 \phi)] \\
& -\frac{i}{2} \sin (2 \chi) \sinh (2 \phi)\left(G_{b b}-G_{a a}\right) .
\end{aligned}
$$

The reason for doing things this way is that $G_{a a}$ and $G_{b b}$ are Lorentzian shapes:

$$
G_{a a}=\frac{1}{\omega-\varepsilon_{a}-i \Gamma_{a}}, \quad G_{b b}=\frac{1}{\omega-\varepsilon_{b}-i \Gamma_{b}},
$$

so we can relate the total spectrum to that obtained from the optical potential model which assumes Lorentzian shapes.

The optical potential model would assume $\Gamma_{a}=\Gamma_{b}=\Gamma$ and $\phi=0$ in Eq. (17). The "exact" expression contains factors $\cosh (2 \phi)$ which modify the intensities of the two components in the spectrum and introduces interference terms $\operatorname{Re}\left(G_{b}-G_{a}\right)$, which changes the spectral shape. In addition the energies $\varepsilon_{a}, \varepsilon_{b}$ and the widths $\Gamma_{a}, \Gamma_{b}$ of the Lorentzian components can be strongly different from those obtained in the optical potential model.

Let us first look more carefully at the Lorentzian contributions and see how they are modified. The energies and widths $\varepsilon_{a, b}$ and $\Gamma_{a, b}$ are determined from a solution of a quadratic complex equation,

$$
\omega_{a, b}=\frac{\varepsilon_{2}+\varepsilon_{1}+i\left(\Gamma_{1}+\Gamma_{2}\right) \pm\left[\left(\varepsilon_{2}-\varepsilon_{1}+i \Delta \Gamma\right)^{2}+4\left(T+i \Gamma_{12}\right)\right]^{1 / 2}}{2},
$$

where $\omega_{a, b}=\varepsilon_{a, b}+i \Gamma_{a, b}$, with $\varepsilon_{a, b}$ being the real parts and $\Gamma_{a, b}$ the imaginary parts.

This shows that both the position and the width of the lines can be influenced by $\Delta \Gamma$ and $\Gamma_{12}$. Of course, if $\Delta \Gamma=\Gamma_{12}=0$ we obtain two lines of equal width. To demonstrate the influence of $\Delta \Gamma$ and $\Gamma_{12}$ on the peak positions and widths we plot in Fig. 2 the peak separation $\left(\varepsilon_{a}-\varepsilon_{b}\right) /\left(\varepsilon_{2}-\varepsilon_{1}\right)$ and the difference in widths $\left(\Gamma_{a}-\Gamma_{b}\right) /\left(\varepsilon_{2}-\varepsilon_{1}\right)$ as a function of $\Delta \Gamma /\left(\varepsilon_{2}-\varepsilon_{1}\right)$ for
$\Gamma_{12}=0$ and as a function of $\Gamma_{12} /\left(\varepsilon_{2}-\varepsilon_{1}\right)$ for $\Delta \Gamma=0$ for various values of $T /\left(\varepsilon_{2}-\varepsilon_{1}\right)$.

For $\Gamma_{12}=0$ and $T=0$ the states $|1\rangle$ and $|2\rangle$ are locally, as well as via the decay processes, fully decoupled. We thus find the trivial result that the difference in decay rate is reflected fully in the difference in width of the pseudostates $|a\rangle$ and $|b\rangle$, while the line splitting is unaffected. This is no longer true if the states $|1\rangle$ and $|2\rangle$ are coupled locally. As can be seen from Fig. $2(a), \Delta \Gamma$ tends to 

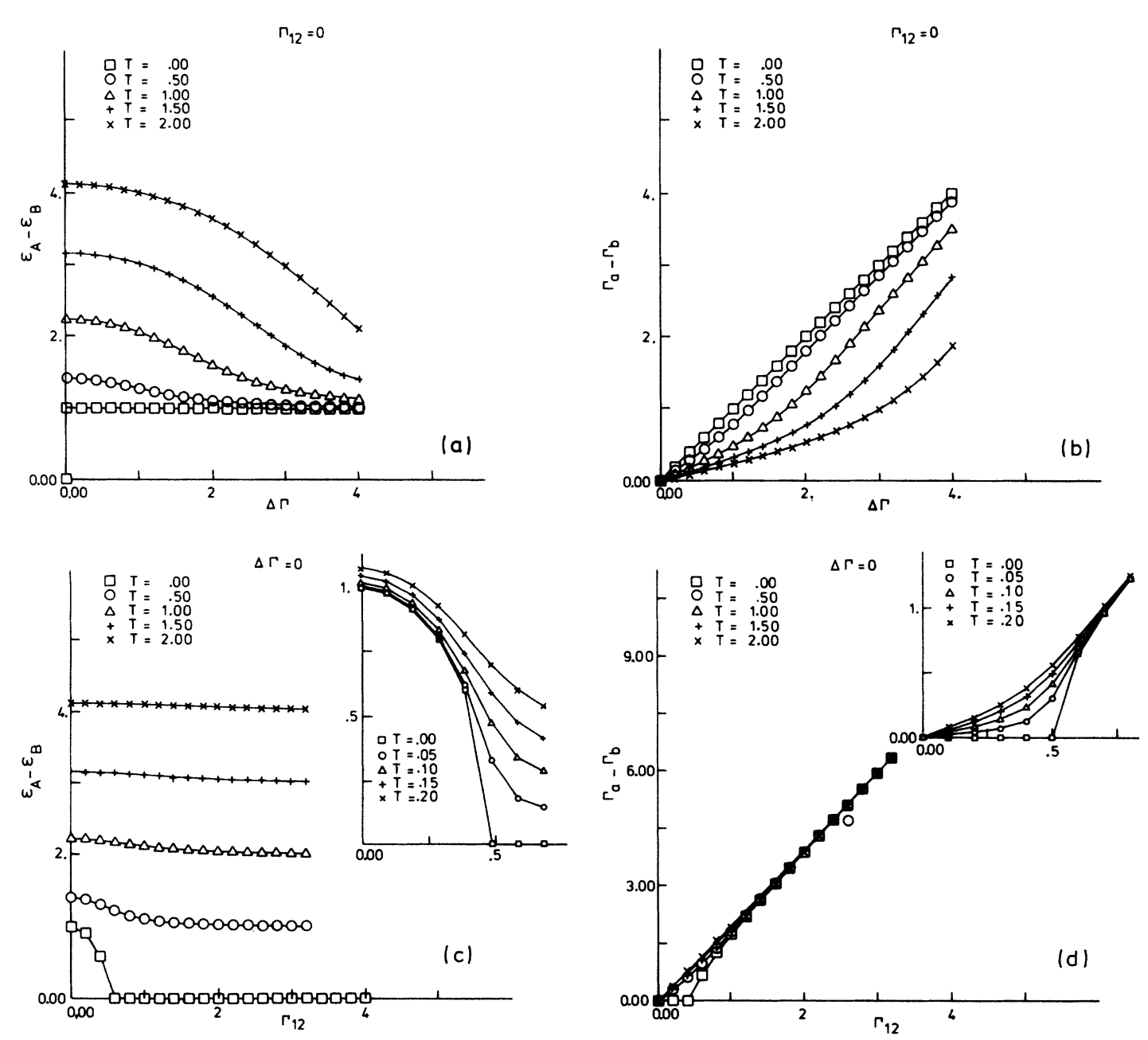

FIG. 2. Difference in splitting [panels (a) and (c)] and width [panels (b) and (d)] of Lorentzian contributions to the line shape as a function of $\Delta \Gamma$ for $\Gamma_{12}=0\left[(a)\right.$ and (b)] and $\Gamma_{12}$ for $\Delta \Gamma=0[(\mathrm{c})$ and (d)] using different values for $T$. Insets of (c) and (d) show large $\varepsilon_{2}-\varepsilon_{1}$ behavior.

decrease the extra line splitting due to $T$. Consider, for instance, the limit $\Delta T \gg 2 T$. It is easily shown that $\omega_{a}-\omega_{b}$ approaches the value $\left(\varepsilon_{2}-\varepsilon_{1}\right)+i \Delta \Gamma$.

From Fig. 2(b) the influence on the width of the lines on $\Delta \Gamma$ can be inferred. As can be seen, the local coupling $T$ has the effective influence to decrease the difference in width. Taking, for instance, $T, \Delta T \gg \varepsilon_{2}-\varepsilon_{1}$, we find, from (19), $\omega_{b}-\omega_{a}=\left(4 T^{2}-\Delta \Gamma^{2}\right)^{1 / 2}$. For this case the width difference in the measurable lines has vanished completely, while the effect of $\Delta \Gamma$ is only responsible for a reduction of the line splitting.

In Figs. 2(c) and 2(d) the same pictures are shown as a function of $\Gamma_{12}$ for $\Delta \Gamma=0$. As can be seen, the behavior is completely different from Figs. 2(a) and 2(b). It is interesting to explore the regime $\Gamma_{12}, T \gg \varepsilon_{2}-\varepsilon_{1}$. We then have, from (19), $\omega_{b}-\omega_{a}=2\left(T+i \Gamma_{12}\right)$. In this limit the line splitting is the same as the splitting found for nondecaying states. On the other hand, the widths of the lines show interesting behavior. The important property of $\Gamma_{12}$ is that it exchanges width between lines in such a way that differences in width are amplified. For instance, it can be shown that $\Gamma_{12}$ is constrained to a maximum value of $\frac{1}{2} \Gamma$ $\left(\Gamma=\Gamma_{1}=\Gamma_{2}\right)$. For this value of $\Gamma_{12}$ we thus find a $\delta$ - function line superposed on a broad line with a width of $2 \times \Gamma$. Note that this behavior is in strong analogy with the well-known phenomenon of exchange narrowing in spin-resonance experiments.

To obtain the core-line XPS spectrum, we must use Eq. (2), which requires knowledge of the transition amplitudes $A_{i}$ from the ground state to the discrete final states. In the sudden approximation for XPS these are given by

$$
A_{i}=\left\langle i \mid c \Psi_{0}\right\rangle,
$$

where $\Psi_{0}$ is the ground-state wave function and $c$ annihilates a core electron. The discrete states $|i\rangle$ which can be reached therefore contain a core hole. Usually in XPS one assumes the core hole to be structureless, so that the states $|i\rangle=|c i\rangle$, where the labels $i$ refer to various possible valence-electron configurations. The only influence of the core hole is to introduce a potential which changes the energetics of the various possible electron configurations relative to the energies in the absence of the core hole. The ground state is, in this model, therefore determined by a linear combination of the same valenceelectron configurations as those describing the discrete states in the presence of the core hole. In our simple 
two-state model we can therefore write

$$
\Psi_{0}=\left(\cos \theta_{0}\right)|1\rangle-\left(\sin \theta_{0}\right)|2\rangle \text {. }
$$

We choose this sign convention because the lowest-energy state in the presence of the core hole is usually the higher-energy state contributing to the ground state because of the core-hole potential. The transition amplitudes of Eq. (2) are then given by $A_{1}=\cos \theta_{0}$ and $A_{2}=-\sin \theta_{0}$, so that

$$
\begin{aligned}
G_{\mathrm{XPS}}= & \left(\cos ^{2} \theta_{0}\right) G_{11}+\left(\sin ^{2} \theta_{0}\right) G_{22} \\
& -2\left(\sin \theta_{0}\right)\left(\cos \theta_{0}\right) G_{12} .
\end{aligned}
$$

Using the relations in Eq. (17), we obtain

$$
G_{\mathrm{XPS}}=C_{a a} G_{a a}+C_{b b} G_{b b}+i C_{a b}\left(G_{b b}-G_{a a}\right),
$$

with

$$
\begin{aligned}
& C_{a a}=\frac{1}{2}\left\{1-\cosh (2 \phi) \cos \left[2\left(\chi-\theta_{0}\right)\right]\right\}, \\
& C_{b b}=\frac{1}{2}\left\{1+\cosh (2 \phi) \cos \left[2\left(\chi-\theta_{0}\right)\right]\right\}, \\
& C_{a b}=\frac{1}{2}\left\{\sinh (2 \phi) \sin \left[2\left(\chi-\theta_{0}\right)\right]\right\},
\end{aligned}
$$

where the angles $\phi$ and $\chi$ are determined by Eq. (17). The XPS spectrum is given by

$$
I_{\mathrm{XPS}}(\omega)=\frac{1}{\pi} \operatorname{Im} G_{\mathrm{XPS}}(\omega) .
$$

We now clearly see the influence of the coupling to the continua. For $\phi=0$, as would be the case for $\Delta \Gamma=\Gamma_{12}=0$ [Eq. (14)], the spectrum consists of two Lorentzian lines of equal width. The influence of nonzero $\Delta \Gamma$ and/or $\Gamma_{12}$ is to transfer intensity between lines at $\varepsilon_{a}$ and $\varepsilon_{b}$ because of the $\cosh (2 \phi)$ factor and to add a dispersivelike contribution given by the last term in Eq. (23). In addition, as discussed above, the peak positions and widths are strongly influenced by $\Delta \Gamma$ and $\Gamma_{12}$

To illustrate this, Eq. (14) is worked out in its real and imaginary parts. For the real and imaginary components of $\theta$ we find

$$
\tan (2 \chi)=\frac{\cosh (2 \phi)(2 T)+\sinh (2 \phi) \delta \Gamma}{\cosh (2 \phi) \Delta \varepsilon-\sinh (2 \phi)\left(2 \Gamma_{12}\right)}
$$

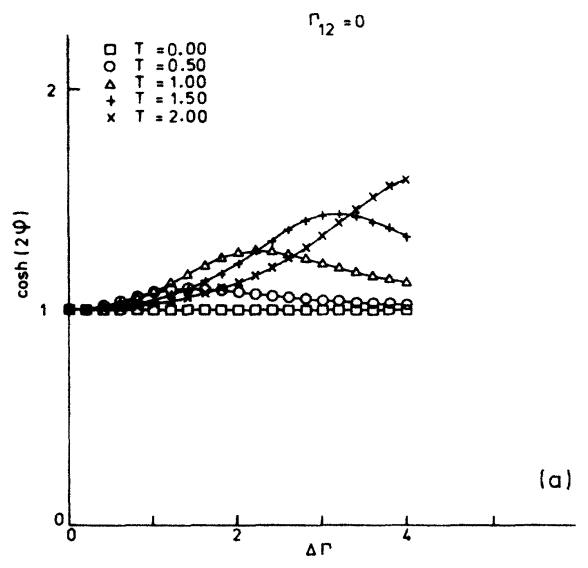

FIG. 3. Measure of interference $\cosh (2 \phi)$ as a function of the same parameters as used in Fig. 1. Divergency as a function of $\Gamma_{12}$ is cut off. and

$$
e^{-8 \phi}=\frac{\left(\Delta \varepsilon+2 \Gamma_{12}\right)^{2}+(\Delta \Gamma-2 T)^{2}}{\left(\Delta \varepsilon-2 \Gamma_{12}\right)^{2}+(\Delta \Gamma+2 T)^{2}},
$$

with $\Delta \varepsilon=\varepsilon_{2}-\varepsilon_{1}$. As can be seen from Eqs. (17) and (26), deviations of $\cosh (2 \phi)$ from 1 are a good measure for the importance of intensity redistribution in-and nonLorentzian contributions to-the spectral line shape. In Fig. 3 we show the behavior of $\cosh (2 \phi)$ in the same parameter range as used in Fig. 2.

For $\Gamma_{12}=0$ [Fig. 3(a)] we see that the deviation of a higher $\Delta \Gamma$ for increasing $T$. The location of the extremum is at

$$
\Delta \Gamma_{\max }=\left[\left(\varepsilon_{2}-\varepsilon_{1}\right)^{2}+4 T^{2}\right]^{1 / 2} .
$$

In the limit $\Delta \Gamma, 2 T \gg \varepsilon_{2}-\varepsilon_{1}, \cosh (2 \phi)$ then has a maximum value $T^{1 / 2}$ at $\Delta \Gamma_{\max }=2 T$. This result shows that the weight of the non-Lorentzian contribution to the line shape $[\sinh (2 \phi)]$ is, in principle, unbounded.

In Fig. 3(b) $\cosh (2 \phi)$ is shown as a function of $\Gamma_{12}$ for $\Delta \Gamma=0$. As for Fig. 2, an interesting limit is $\Gamma_{12}, T \gg \varepsilon_{2}-\varepsilon_{1}$. As can be seen, $\cosh (2 \phi)$ is very near to 1 , which leads us to conclude that in the fluctuationdominated regime the effect of $\Gamma_{12}$ is mainly the aforementioned "exchange narrowing."

In Fig. 4 we have shown several calculated spectra to demonstrate the influence of $\Delta \Gamma$ and $\Gamma_{12}$. The spectra have been decomposed into the various contributions from $G_{a a}, G_{b b}$, and $G_{a b}$. As parameters, we have taken $\varepsilon_{2}-\varepsilon_{1}=1 \mathrm{eV}$ in the presence of the core hole and $\varepsilon_{2}-\varepsilon_{1}=-1 \mathrm{eV}$ in the initial state.

On the extreme left-hand side of Fig. 4 are the spectra corresponding to $\Delta \Gamma=\Gamma_{12}=0$ for various values of $T$. These would correspond to the optical potential approximation. Figure $4(b)$ shows the result of different decay rates for the two states $(\Delta \Gamma=-1)$ exhibiting a strong distortion of the spectra. In fact, there is little resemblance to the optical approximation. In Fig. 4(c) we show the influence of the coupling to the same continuum $\left(\Gamma_{12}=-1\right)$ for $\Delta \Gamma=0$ and the same values of $T$ as used in Fig. 4(a). This shows the strong dependence of the line shape on

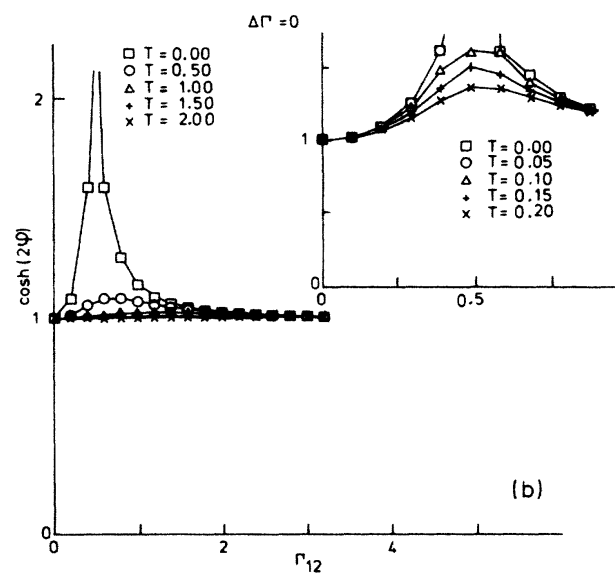
Lorentzian line shape has an extremum, which shifts to 


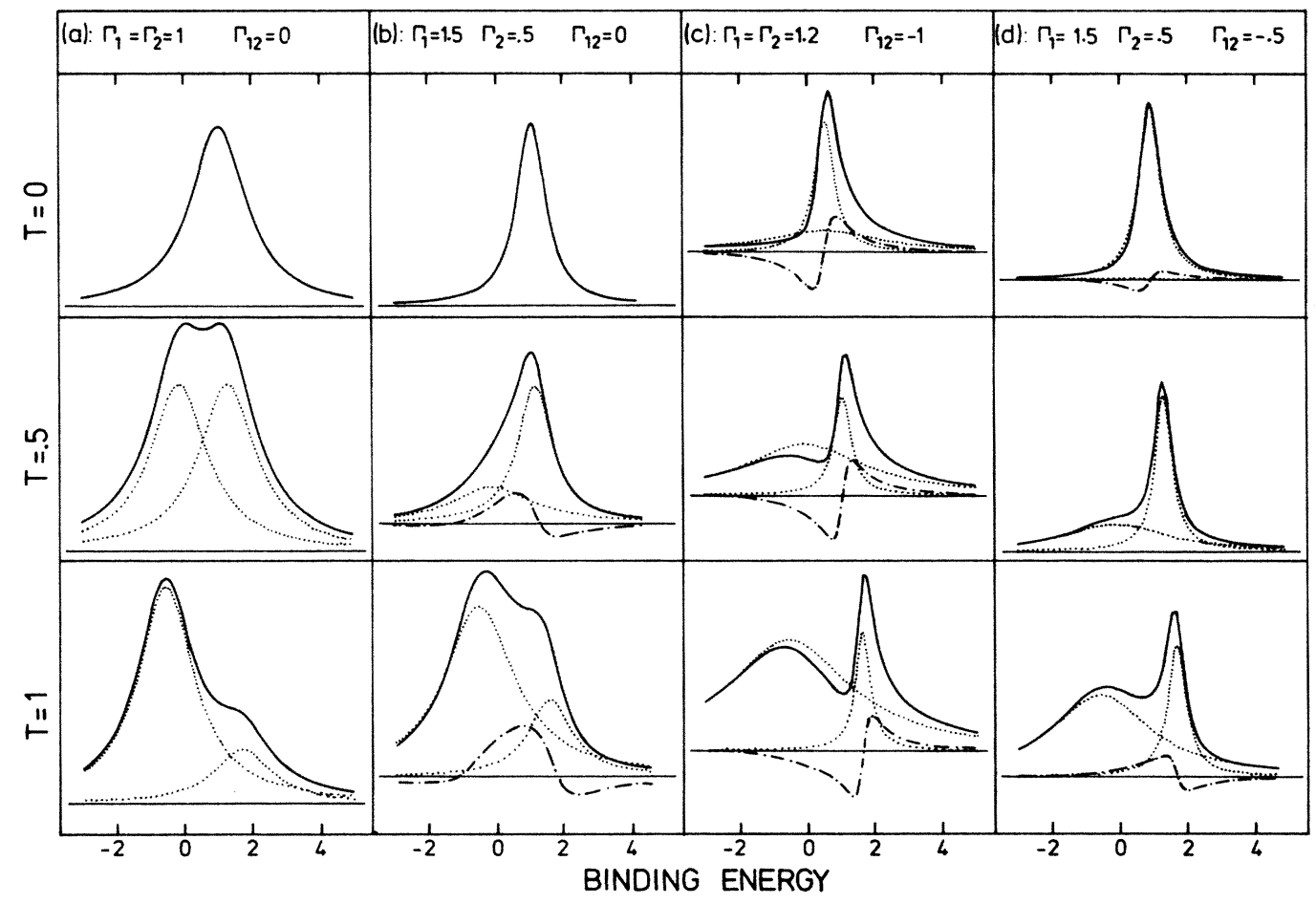

FIG. 4. XPS spectra of the two-level model as a function of $T$ for various values of $\Gamma_{12}$ and $\Gamma$. Straight line is the total XPS spectrum, dotted lines show the Lorentzian contributions, and dashed-dotted line shows the sum of the dispersionlike contributions.

$\Gamma_{12}$, especially for large $T$. Finally, in Fig. 4(d) we show the combined influence of $\Delta \Gamma$ and $\Gamma_{12}$ on the spectrum.

\section{APPLICATION TO XPS CHARGE-TRANSFER SATELLITES}

Having shown the possibly strong dependence of the XPS core line shape and relative component intensities on the details of the coupling to continua, we must now see for which core lines and materials we expect to get substantial values for $\Delta \Gamma$ and/or $\Gamma_{12}$. First of all, we note that $\Delta \Gamma$ and/or $\Gamma_{12}$ since they involve core-hole decay processes will usually not be larger than several $\mathrm{eV}$. For example, in the $3 d$ holes in the rare-earth metals or the $2 p$ holes in transition metals the total lifetime broadenings are between 0.5 and $2 \mathrm{eV}$. From the foregoing discussion, we see that a substantial influence of $\Delta \Gamma$ and/or $\Gamma_{12}$ is only expected for final-state energy separations less than about $10 \mathrm{eV}$. The spectra which can be influenced then are those involving the same core hole, but with differing valence-electron configuration, as for the screened and unscreened peaks observed in $3 d$ photoemission of Ce compounds ${ }^{3,4,6,7}$ and the $2 p$ satellite lines in $3 d$-transitionmetal compounds. $1,2,8-18,28$ The question remaining then is that concerning the physical origin and magnitude of $\Delta \Gamma$ and $\Gamma_{12}$.

To see how these terms enter, consider the general problem of satellite spectra due to charge transfer. For the sake of simplicity, we take a cluster approach, which has been shown to be quite accurate for at least insulating strongly correlated materials. ${ }^{1-3,28}$ In these models a cluster consisting of a metal ion surrounded by ligands is solved by configuration interaction. The ground state of the cluster can be written as a linear combination of states $\left|d^{n} L^{m}\right\rangle,\left|d^{n+1} L^{m-1}\right\rangle$, etc. In the presence of a core hole the energy of these states is lowered, proportional to the number of $d$ electrons they contain, due to the corehole- $d$-electron interaction. New eigenstates are found which are assigned to the satellite structure. Relating to our general treatment in the preceding section, the states $\left|d^{n} L^{m}\right\rangle$ are the discrete states which are coupled $\left(\left\langle d^{n} L^{m}|H| d^{n+1} L^{m-1}\right\rangle=T\right)$.

Next we consider the decay channels. As an example we take an $L M V$ Auger channel (the argument applies equally well to $L V V$, etc.). Starting from a state $\left|\underline{2 p} d^{n} L^{m}\right\rangle$, a state like $\left|\underline{3 p} d^{n-1} L^{m} k\right\rangle$ ( $k$ denoting a continuum electron) is reached. These continuum states, however, cannot be directly identified with those given in Eq. (1) since the continuum states $\left|3 p d^{n-1} L^{m} k\right\rangle$ and $\left|3 p d^{n} L^{m-1} k\right\rangle$ are coupled. Neglecting the $k$ dependence of the coupling, this will be given also by

$$
\left\langle\underline{3 p} d^{n-1} L^{m} k|H| \underline{3 p} d^{n} L^{m-1} k\right\rangle \propto T .
$$

If we first diagonalize the continuum part of the spectrum, we find, in the case of two continua,

$$
\begin{aligned}
& \Psi_{k 1}=\left(\cos \theta_{c}\right)\left|\underline{3 p} d^{n-1} L^{m} k\right\rangle+\left(\sin \theta_{c}\right)\left|\underline{3 p} d^{n} L^{m-1} k\right\rangle, \\
& \Psi_{k 2}=\left(-\sin \theta_{c}\right)\left|\underline{3 p} d^{n-1} L^{m} k\right\rangle+\left(\cos \theta_{c}\right)\left|\underline{3 p} d^{n} L^{m-1} k\right\rangle,
\end{aligned}
$$

in which $\theta_{c}$ are determined by the hybridization of the Auger final state.

We now see that the discrete states $\left|\underline{2 p} d^{n} L^{m}\right\rangle(|1\rangle)$ and $\left|2 p d^{n+1} L^{m-1}\right\rangle(|2\rangle)$ supposed to be reached in the photoemission process couple to both continua $\Psi_{k 1}$ and 
$\Psi_{k 2}$. Taking

$$
\left\langle 1|H| \underline{3 p} d^{n-1} L^{m} k\right\rangle=\left\langle 2|H| \underline{\left.3 p d^{n} L^{m-1} k\right\rangle=V_{k},}\right.
$$

we find from Eq. (28), for the $V_{k_{n} i}$ 's appearing in Eq. (1),

$$
\begin{aligned}
& V_{k_{1} 1}=\left(\cos \theta_{c}\right) V_{k}, \quad V_{k_{1} 2}=\left(\sin \theta_{c}\right) V_{k}, \\
& V_{k_{2} 1}=-\left(\sin \theta_{c}\right) V_{k}, \quad V_{k_{2} 2}=\left(\cos \theta_{c}\right) V_{k} .
\end{aligned}
$$

Inserting these into Eq. (10) yields, for $\Gamma_{1}, \Gamma_{2}$, and $\Gamma_{12}$,

$$
\begin{aligned}
& \Gamma_{1}=\left(\cos ^{2} \theta_{c}\right) \rho\left(\omega-\varepsilon_{k 1}\right)+\left(\sin ^{2} \theta_{c}\right) \rho\left(\omega-\varepsilon_{k 2}\right), \\
& \Gamma_{2}=\left(\sin ^{2} \theta_{c}\right) \rho\left(\omega-\varepsilon_{k 1}\right)+\left(\cos ^{2} \theta_{c}\right) \rho\left(\omega-\varepsilon_{k 2}\right), \\
& \Gamma_{12}=\left(\cos \theta_{c}\right)\left(\sin \theta_{c}\right)\left[\rho\left(\omega-\varepsilon_{k 1}\right)-\rho\left(\omega-\varepsilon_{k 2}\right)\right],
\end{aligned}
$$

in which $\varepsilon_{k 1}$ and $\varepsilon_{k 2}$ are the eigenenergies of the continuum states $\Psi_{k 1}$ and $\Psi_{k 2}$ and

$$
\rho\left(\omega-\varepsilon_{k i}\right)=\operatorname{Im}\left[\sum_{k} \frac{V_{k}^{2}}{\omega-\varepsilon_{k i}}\right] \text {. }
$$

From Eq. (30) we see that if $\rho\left(\omega-\varepsilon_{k 1}\right)$ is unequal to $\rho\left(\omega-\varepsilon_{k 2}\right)$, we generally expect that the $\Delta \Gamma$ as well as the $\Gamma_{12}$ are unequal to zero. This condition will be met for near-threshold decay processes. Because the thresholds of continua $\Psi_{k 1}$ and $\Psi_{k 2}$ are, in general, nondegenerate due to the energy stored in the system after Auger emission, evaluation of Eq. (31) at the positions of the discrete states which are reached in photoemission will yield different values for $\rho\left(\omega-\varepsilon_{k_{1}}\right)$ and $\rho\left(\omega-\varepsilon_{k_{2}}\right)$, as long as the density of states of the emitted Auger electron is not constant. This condition is certainly met for the Coster-Kronig decay of a $2 p_{1 / 2}$ hole in $3 d$ transition metals, as we will show in detail in the next section; strong interference effects can then be expected.

On the other hand, if the Auger process is far away from threshold, the density of states of the emitted electron will be essentially constant over at least the energy range of the XPS spectrum. As can be seen from Eq. (30), we retain in this case the "optical potential approximation." In the Appendix we generalize this theory to many locally coupled continua. it appears that for this case $\Delta \Gamma$ and $\Gamma_{i j}$ also vanish far from threshold.

As far as valence-band charge-fluctuation processes are concerned, this seems to be a general rule. Only under the special condition of scarcity of valence electrons it can happen that a $K L V$ or $K V V$ Auger decay process of a particular valence-electron configuration becomes impossible, giving rise to $\Delta \Gamma$-like effects in the XPS spectrum as shown by Gunnarsson and Schönhammer. ${ }^{25}$ At least for the late $3 d$ transition metals this effect will be unimportant.

\section{APPLICATION: THE $2 p_{1 / 2}$ SPECTRUM OF $\mathrm{NiBr}_{2}$}

In order to show that the Coster-Kronig decay rates have the proper order of magnitude to cause the large differences between $2 p_{1 / 2}$ and $2 p_{3 / 2}$ line shapes of transition-metal compounds, the special case of $\mathrm{NiBr}_{2} 2 p$ XPS (Fig. 6) is worked out.

As we showed recently, ${ }^{28}$ the $2 p_{3 / 2}$ XPS lines of the Ni dihalides can be well understood within the framework of the cluster approximation. One assumes that the ground state can be approximated by the ground state of a $\left(\mathrm{NiL}_{6}\right)^{4-}$ cluster, ${ }^{28}$

$$
\Phi_{0}=\alpha_{0}\left|d^{8}\right\rangle+\beta_{0}\left|d^{9} \underline{L}\right\rangle+\gamma_{0}\left|d^{10} \underline{L}^{2}\right\rangle,
$$

where $\underline{L}$ denotes a hole in the anion $p$ orbital.

This wave function has to be determined by solving a Hamiltonian matrix with the nonzero matrix elements

$$
\begin{aligned}
& \left\langle d^{8}|H| d^{8}\right\rangle=0, \\
& \left\langle d^{9} \underline{L}|H| d^{9} \underline{L}\right\rangle=\Delta, \\
& \left\langle d^{10} \underline{L}{ }^{2}|H| d^{10} \underline{L}^{2}\right\rangle=2 \Delta+U, \\
& \left\langle d^{8}|H| d^{9} \underline{L}\right\rangle=\left\langle d^{9} \underline{L}|H| d^{10} \underline{L}^{2}\right\rangle=\sqrt{2} T,
\end{aligned}
$$

$\Delta$ being the energy cost to transfer a valence hole from $\mathrm{Ni}$ to the ligands, $U$ the mutual Coulomb repulsion of two electrons centered on the $\mathrm{Ni}$ ion, and $T$ the hybridization interaction.

After core ionization the valence electrons on $\mathrm{Ni}$ are stabilized with an amount $Q$ because of their Coulomb interaction with the core hole, while the hybridization rates are assumed to be unaffected by the presence of the core hole,

$$
\begin{aligned}
& \left\langle\underline{2 p}_{3 / 2} d^{8}|H| \underline{2 p} \underline{p}_{3 / 2} d^{8}\right\rangle=\varepsilon_{2 p_{3 / 2}}, \\
& \left\langle\underline{2 p}_{3 / 2} d^{9} \underline{L}|H| \underline{2 p}_{3 / 2} d^{9} \underline{L}\right\rangle=\varepsilon_{2 p_{3 / 2}}+\Delta-Q, \\
& \left\langle\underline{2 p}_{3 / 2} d^{10} \underline{L}^{2}|H| \underline{2 p}_{3 / 2} d^{10} \underline{L}\right\rangle=\varepsilon_{2 p_{3 / 2}}+2(\Delta-Q)+U, \\
& \left\langle\underline{2 p}_{3 / 2} d^{8}|H| \underline{2 p}_{3 / 2} d^{9} \underline{L}\right\rangle=\left\langle\underline{2 p}_{3 / 2} d^{9} \underline{L}|H| \underline{2 p} \underline{p}_{3 / 2} d^{10} \underline{L}^{2}\right\rangle \\
& =\sqrt{2} T .
\end{aligned}
$$

As we showed, ${ }^{28}$ the quantitative analysis of these $2 p_{3 / 2}$ satellite spectra yields quite accurate results for the ground-state properties for all $\mathrm{Ni}$ dihalides. The fit of the $\mathrm{NiBr}_{2}$ spectrum using $\Delta=3 \mathrm{eV}, U=5 \mathrm{eV}, Q=7 \mathrm{eV}$, and $T=2 \mathrm{eV}$, and convoluted with a Gaussian and a Lorentzian to mimic instrumental, lifetime, and multiplet broadenings is shown in Fig. 6(a).

For the $2 p_{1 / 2}$ spectrum, we incorporate the CosterKronig decay in the way explained in Sec. III. We have the Coster-Kronig (CK) channels

$$
\begin{aligned}
& \left|\underline{2 p}_{1 / 2} d^{8}\right\rangle{ }^{v_{\mathrm{CK}}}\left|\underline{2 p}_{3 / 2} d^{7} k\right\rangle, \\
& \left|\underline{2 p}_{1 / 2} d^{9} \underline{L}\right\rangle \stackrel{{ }^{v_{\mathrm{CK}}}}{\rightarrow}\left|\underline{2 p}_{3 / 2} d^{8} \underline{L} k\right\rangle, \\
& \left|\underline{2 p}_{1 / 2} d^{10} \underline{L}^{2}\right\rangle \stackrel{v_{\mathrm{CK}}}{\rightarrow}\left|\underline{2 p}_{3 / 2} d^{9} \underline{L}^{2} k\right\rangle,
\end{aligned}
$$

in which $k$ denotes a continuum electron in the conduction bands. Aside form the additional Coster-Kroning decay channels, the $2 p_{1 / 2}$ XPS states are assumed to be governed by the Hamiltonian matrix given in Eq. (34). The location of the Coster-Kronig final states on the right-hand side of (35) can be estimated with some accuracy. The Coster-Kronig final states resemble the valenceband spectrum in the presence of a $2 p_{3 / 2}$ hole and a $4 s p$ cleciruir. Taking the energy of $\left|\underline{2 p}_{1 / 2} d^{8}\right\rangle$ as the zero 
point, we then find

$$
\begin{gathered}
\left\langle\underline{2 p}_{3 / 2} d^{7} k|H| \underline{2 p}_{3 / 2} d^{7} k\right\rangle=\varepsilon_{L}-\frac{3}{2} \alpha+\varepsilon_{k}-(\Delta-Q)+U, \\
\left\langle\underline{2 p}_{3 / 2} d^{8} \underline{L} k|H| \underline{2 p}_{3 / 2} d^{8} \underline{L} k\right\rangle=\varepsilon_{L}-\frac{3}{2} \alpha+\varepsilon_{k}, \\
\left\langle\underline{2 \underline{p}}_{3 / 2} d^{9} \underline{L}^{2} k|H| \underline{2 p}_{3 / 2} d^{9} \underline{L}^{2} k\right\rangle=\varepsilon_{L}-\frac{3}{2} \alpha+\varepsilon_{k}+(\Delta-Q), \\
\left\langle\underline{2}_{3 / 2} d^{10} \underline{L}^{3}|H| \underline{2 p}{ }_{3 / 2} d^{10} \underline{L}^{3} k\right\rangle=\varepsilon_{L}-\frac{3}{2} \alpha+\varepsilon_{k} \\
+2(\Delta-Q)+U,
\end{gathered}
$$

in which $\frac{3}{2} \alpha$ is the spin-orbit splitting $(\sim 18.0 \mathrm{eV}), \varepsilon_{L}$ the energy it costs to create a ligand hole, and $\varepsilon_{k}$ the energy of an $s p$ electron.

Taking into account the degeneracy of the CosterKronig final state as for the UPS spectrum ${ }^{29}$ and neglecting $k$ dependence, the hybridization of the Coster-Kronig states is given by

$$
\begin{aligned}
& \left\langle\underline{2 \underline{p}}_{3 / 2} d^{7} k|H| \underline{2 p}_{3 / 2} d^{8} \underline{L} k^{\prime}\right\rangle \\
& =\left\langle\underline{2 p}_{3 / 2} d^{9} \underline{L} k|H| \underline{2 p}_{3 / 2} d^{10} \underline{L}^{3} k^{\prime}\right\rangle \\
& =\sqrt{3} T \delta_{k k^{\prime}}
\end{aligned}
$$

The energetics of the Coster-Kronig final state is now fully determined, except for the factor $\varepsilon_{L}+\varepsilon_{k=0}-\frac{3}{2} \alpha$, which determines the position of the edge of the continuum $\left|2 \underline{2}_{3 / 2} d^{8} \underline{L} k\right\rangle$ with respect to the state $\left|2 p_{1 / 2} d^{8}\right\rangle$. This can be determined from the recent $x$-ray-absorption spectroscopy (XAS) data of van der Laan et al. ${ }^{30}$ These spectra show white lines which are interpreted in the cluster approximation as

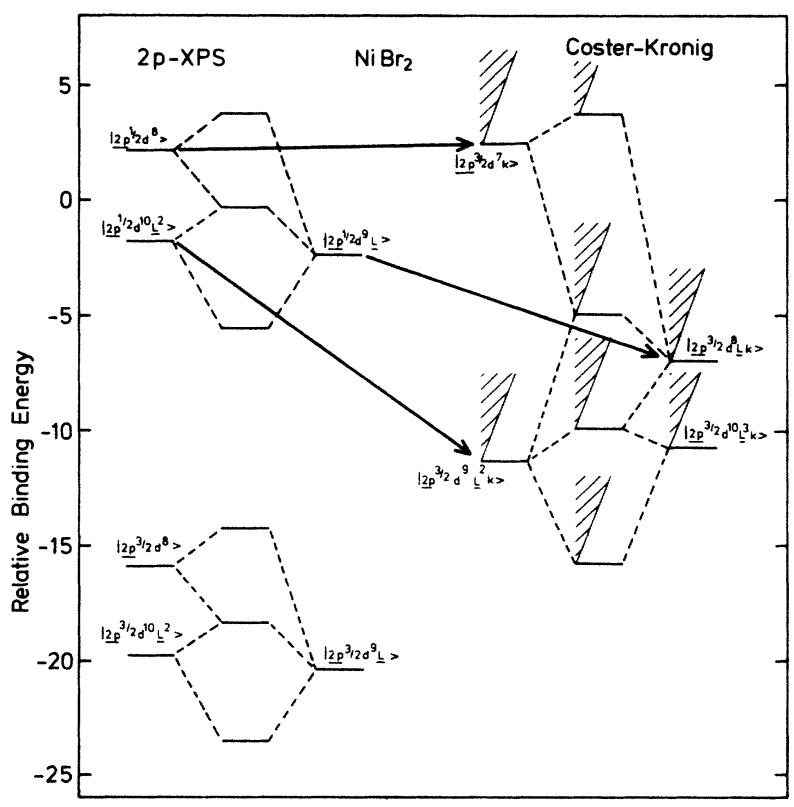

FIG. 5. Level scheme of the discrete XPS states and the Coster-Kronig continua as found for the $2 p$ XPS of $\mathrm{NiBr}_{2}$. Straight lines on the right-hand side indicate continuum thresholds and shaded areas simulate the uprise of the $4 s p$ density of states.

$$
\begin{aligned}
& \left|d^{8}\right\rangle \stackrel{\hbar \omega}{\rightarrow}\left|\underline{2 p} d^{9}\right\rangle, \\
& \left|d^{9} \underline{L}\right\rangle \stackrel{\hbar \omega}{\rightarrow}\left|\underline{2 p} d^{10} \underline{L}\right\rangle .
\end{aligned}
$$

Also, continuum absorptions are observed which are assigned to absorptions into the $\mathrm{Ni} 4 s p$ continua accompanied by relaxation from $d$-channel electrons,

$$
\begin{aligned}
& \left|d^{8}\right\rangle \stackrel{\hbar \omega}{\rightarrow}\left|\underline{2 p} d^{8} k\right\rangle, \\
& \left|d^{9} \underline{L}\right\rangle \stackrel{\hbar \omega}{\rightarrow}\left|\underline{2 p} d^{9} \underline{L} k\right\rangle, \\
& \left|d^{10} \underline{L}^{2}\right\rangle \stackrel{\hbar \omega}{\rightarrow}\left|\underline{2 p} d^{10} \underline{L}^{2} k\right\rangle .
\end{aligned}
$$

From the position of the $2 p_{3 / 2}$ continuum edge with respect to the $2 p_{1 / 2}$ white lines, the energy difference between $\left|2 p_{1 / 2} d^{9}\right\rangle$ and $\left|2 p_{3 / 2} d^{9} \underline{L} k\right\rangle$ can be determined, which equals the unknown energy $\varepsilon_{L}+\varepsilon_{k=0}-\frac{3}{2} \alpha$. van der Laan et al. ${ }^{30}$ find for this quantity $\approx-9.0 \mathrm{eV}$.

In Fig. 5 we show the level scheme using the foremen-

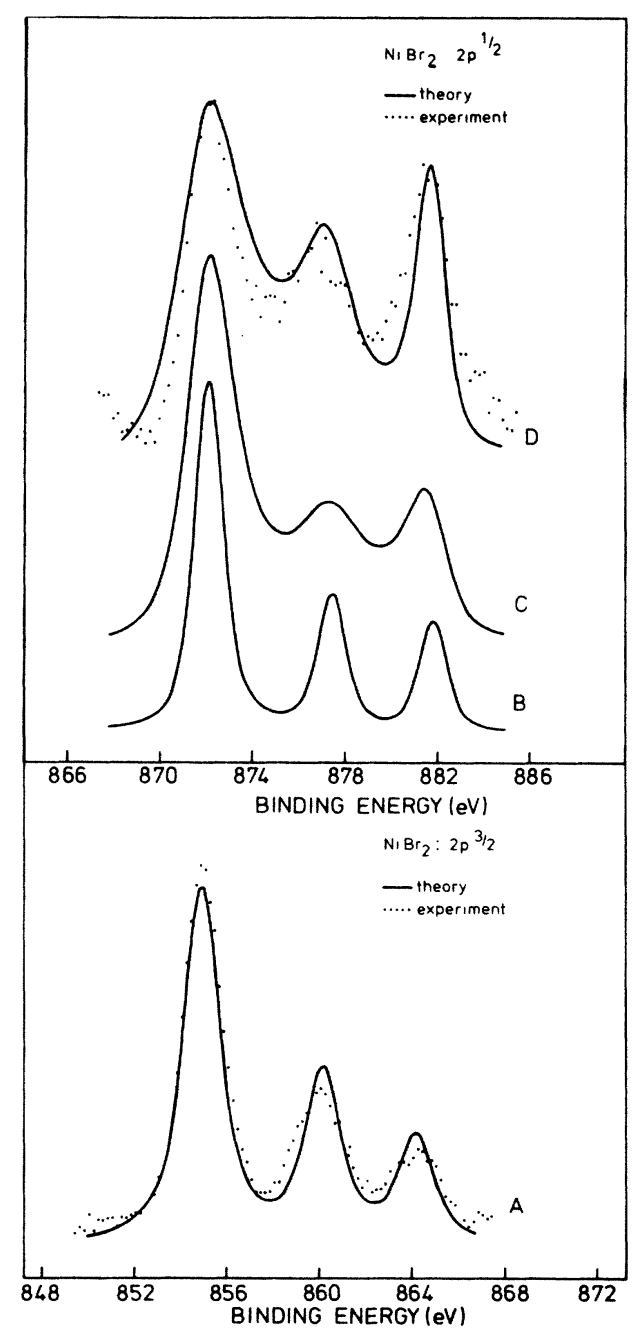

FIG. 6. Experimental and theoretical $2 p$ XPS spectra of $\mathrm{NiBr}_{2}$. In (a) the $2 p_{3 / 2}$ spectrum is shown and in (d) the $2 p_{1 / 2}$ spectrum with the theoretical line obtained from the full theory. (b) and (c), respectively, show theoretical results for no imaginary parts and surpressed $\operatorname{Im} \Gamma_{i j}, i \neq j$. 
tioned parameters. From this figure it can be seen that the Coster-Kronig process will influence the XPS line shape. The $\left|2 p_{1 / 2} d^{8}\right\rangle$ state is even beneath its "own" continuum and is only allowed to decay via the mixing of $\left|2 p_{3 / 2} d^{7} k\right\rangle$ character in the other continua. On the other hand, $\left|2 p_{1 / 2} d^{10} \underline{L}^{2}\right\rangle$ will decay rapidly, while $\left|2 p_{1 / 2} d^{9} \underline{L}\right\rangle$ lies somewhere in between. Moreover, although the energy spread of the Coster-Kronig basis states is large, the hybridization matrix elements are also large, yielding strongly mixed continua.

In Fig. 6(d) we show the result of a numerical calculation of the $2 p_{1 / 2}$ spectrum using the method explained in the Appendix. Only the shape of the $s p$ continuum and the strength of the Coster-Kronig matrix element are adjustable parameters in the calculation, although they are together constrained by the integrated width in the spectrum. We used a density of states which linearly increases up to $10 \mathrm{eV}$ above threshold and is constant hereafter. In the flat region the decay strength $\pi V_{\text {CK }}^{2} \rho$ equals $2 \mathrm{eV}$.

In order to give an impression of the relative importance of the various contributions to the line shape, we show in Fig. 6(b) the spectrum obtained by taking only the real parts of $\varepsilon_{i}^{\prime}$ and $T_{i j}^{\prime}$ in Eq. (9) into account and an equal linewidth for all components. This looks much like the $2 p_{3 / 2}$ spectrum, showing that the influence of the continua on the real parts is small. In Fig. 6(c) we add the imaginary parts of $\varepsilon_{i}^{\prime}$, which are different for the various final states. We still see a spectrum qualitatively similar to the $2 p_{3 / 2}$ spectrum. In Fig. $6(\mathrm{~d})$ is shown the calculated spectrum taking all effects into account. This agrees very well with the $2 p_{1 / 2}$ experimental spectrum shown as dots. This shows that for these systems the $\operatorname{Im} \Gamma_{i j}^{\prime}$ is the dominant cause of strong distortion in the $2 p_{1 / 2}$ spectrum.

\section{CONCLUSIONS}

In this paper we have shown that it is important to include the details of decay processes in XPS line-shape analysis if the continua involved are close to threshold. We have presented a general theory for doing this and have shown that the line-shape difference between the $2 p_{3 / 2}$ and $2 p_{1 / 2}$ regions of the XPS spectra of $\mathrm{Ni}$ dihalides can be understood, provided one includes the details of the Coster-Kronig decay continua.

Since, however, the details of the decay continua are often not known, one should try to avoid using XPS core states with near-threshold decay continua in the analysis of line shapes in terms of model Hamiltonian parameters.

\section{ACKNOWLEDGMENTS}

The authors thank D. van der Marel and G. van der Laan for helpful discussions. This investigation was supported by the Netherlands Foundation for Chemical Research [Stichting Organisatie voor Zuiver-Wetenschappelÿk Onderzoek (SON)] with financial aid from the Netherlands Organization for the Advancement of Pure Research [Nederlandse Organisatie voor ZuiverWetenschappelÿk Onderzoek (ZWO)].

\section{APPENDIX}

Here the example of two coupled states and two coupled continua is generalized to many coupled states and many coupled continua. The easiest way to do this is by defining a projector operator projecting out the discrete states,

$$
P=\sum_{l}\left|\underline{2 p} d^{n+l} L^{m-l}\right\rangle\left\langle\underline{2 p} d^{n+l} L^{m-l}\right| .
$$

From the well known identity $(Q=1-P)$,

$$
P \frac{1}{z-H} P=\left[z P-P H P-P H Q \frac{1}{z-Q H Q} Q H P\right)^{-1},
$$

we find that the XPS spectrum is found by inverting a pseudo-Hamiltonian with diagonal elements,

$$
\left\langle\underline{2 p} d^{n+l} L^{m-l}|z-H| \underline{2 p} d^{n+l} L^{m-l}\right\rangle=z-E\left(\underline{2 p} d^{n+l} L^{m-l}\right)-\sum_{k} V_{k}^{2}\left\langle\underline{3 p} d^{n+l-1} L^{m-l} k\left|\frac{1}{z-\widetilde{H}}\right| \underline{3 p} d^{n+l-1} L^{m-l} k\right\rangle,
$$

and nondiagonal elements

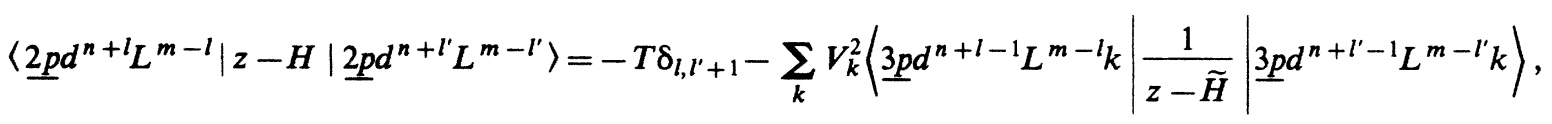

in which we defined $\widetilde{H}=Q H Q$, which thus only acts on the Auger states. Because the dynamics contained in $\widetilde{H}$ is $k$ independent, it makes sense to define a transformation which diagonalizes $\widetilde{H}$,

$$
\underline{U}^{-1} \underline{\widetilde{H}} \underline{U}=\underline{\Lambda} \text {. }
$$

The decay self-energies appearing in Eqs. (A3) and (A4) can be rewritten using (A5),

$$
\begin{array}{r}
\sum_{k} V_{k}^{2}\left\langle\underline{3 p} d^{n+l-1} L^{m-l} k\left|\frac{1}{z-\widetilde{H}}\right| \underline{3 p} d^{n+l^{\prime}-1} L^{m-l^{\prime}} k\right\rangle \\
=\sum_{l} u_{i l}^{-1} u_{l j} \sum_{k} \frac{V_{k}^{2}}{z-\lambda_{l}-\varepsilon_{k}},
\end{array}
$$

with

$$
|i\rangle=\left|\underline{3 p} d^{n+l-1} L^{m-l} k\right\rangle
$$


and

$$
|j\rangle=\left|\underline{3 p} d^{n+l^{\prime}-1} L^{m-1^{\prime}} k\right\rangle,
$$

while $\lambda_{l}$ denotes the $l$ th eigenvalue of $\widetilde{H}$ and $u_{i j}{ }^{-1}, u_{i j}$ are matrix elements of $\underline{U}^{-1}$ and $\underline{U}$.

Equation (A6) is the result which we used in the calculations of Sec. IV. Also, the validity regime of the optical potential approximation can be easily found from (A6). We argued that far away from threshold the continuum electron density of states (and $V_{k}^{2}$ ) will be essentially constant $(i \Gamma$ ). From Eq. (A6) we find

$$
\sum_{l} u_{i l}^{-1} u_{l j} \sum_{k} \frac{V_{k}^{2}}{z-\lambda_{p}-\varepsilon_{k}}=\sum_{l} i u_{i l}^{-1} u_{l j} \Gamma=i \Gamma \delta_{i j},
$$

which shows that the optical potential approximation is valid for processes far away from threshold, independent of the number of coupled Auger continua.
${ }^{1}$ G. van der Laan, C. Westra, C. Haas, and G. A. Sawatzky, Phys. Rev. B 23, 4369 (1981).

2S. Larsson, Chem. Phys. Lett. 40, 362 (1976).

${ }^{3}$ A. Fujimori, Phys. Rev. B 27, 3992 (1983); 28, 2281 (1983); 28, 4489 (1983).

${ }^{4}$ A. Kotani, H. Mizuta, T. Jo, and J. C. Parlebas, Solid State Commun. 53, 805 (1985).

${ }^{5}$ D. K. G. de Boer, C. Haas, and G. A. Sawatzky, Phys. Rev. B 29, 4401 (1984).

${ }^{6} \mathrm{O}$. Gunnarsson and K. Schönhammer, Phys. Rev. Lett. 50, 604 (1983); Phys. Rev. B 28, 4315 (1983).

7J. C. Fuggle, F. U. Hillebrecht, Z. Zolnieriek, R. Lasser, Ch. Freiburg, O. Gunnarsson, and K. Schönhammer, Phys. Rev. B 27, 7330 (1983).

${ }^{8}$ A. Rosencwaig, G. K. Wertheim, and H. J. Guggenheim, Phys. Rev. Lett. 27, 479 (1971).

${ }^{9}$ D. C. Frost, C. A. McDowell, and I. S. Woolsey, Chem. Phys. Lett. 17, 320 (1972).

${ }^{10}$ L. J. Matienzo, L. I. Yin, S. O. Grim, and W. E. Swartz, Inorg. Chem. 12, 2762 (1973).

${ }^{11}$ T. A. Carlson, J. C. Carver, L. J. Saethre, F. Garcia Santibanez, and G. A. Vernon, J. Electron Spectrosc. Relat. Phenom. 5, 247 (1974).

12T. A. Carlson, J. C. Carver, and G. A. Vernon, J. Chem. Phys. 62, 932 (1975).

${ }^{13}$ G. A. Vernon, G. Stucky, and T. A. Carlson, Inorg. Chem. 15, 278 (1976).

${ }^{14}$ M. A. Brisk and A. D. Baker, J. Electron Spectrosc. Relat.
Phenom. 6, 81 (1975).

${ }^{15}$ B. Wallbank, I. G. Main, and C. E. Johnson, J. Electron Spectrosc. Relat. Phenom. 5, 259 (1974).

${ }^{16}$ K. S. Kim, Phys. Rev. B 11, 2177 (1975).

${ }^{17}$ M. Scrocco, J. Electron Spectrosc. Relat. Phenom. 19, 311 (1980).

18B. W. Veal and A. P. Paulikas, Phys. Rev. B 31, 5399 (1985).

${ }^{19}$ R. P. Gupta and S. K. Sen, Phys. Rev. B 12, 15 (1975).

${ }^{20}$ R. Rekha, S. Pal, and R. P. Gupta, Phys. Rev. B 26, 35 (1982).

${ }^{21}$ S. Asada, C. Satoka, and S. Sugano, J. Phys. Soc. Jpn. 38, 855 (1975).

22S. Asada and S. Sugano, J. Phys. Soc. Jpn. 41, 1291 (1976).

${ }^{23}$ S. P. Kowalczyk, L. Ley, F. R. McFeely, and D. A. Shirley, Phys. Rev. B 11, 1721 (1975).

${ }^{24} \mathrm{G}$. Wendin, Struct. Bonding (Berlin) 45, 1 (1981).

${ }^{25}$ O. Gunnarsson and K. Schönhammer, Phys. Rev. B 22, 3710 (1980).

${ }^{26}$ L. C. Davis and L. A. Feldkamp, Solid State Commun. 19, 413 (1976); Phys. Rev. B 15, 2961 (1977).

27U. Fano, Phys. Rev. 124, 1866 (1961).

28J. Zaanen, C. Westra, and G. A. Sawatzky, preceding paper, Phys. Rev. B 33-I, 8060 (1986).

29J. Zaanen, G. A. Sawatzky, and J. W. Allen, J. Magn. Magn. Mater. 54-57, 607 (1986).

${ }^{30}$ G. van der Laan, J. Zaanen, and G. A. Sawatzky, Solid State Commun. 56, 673 (1985); Phys. Rev. B 33, 4253 (1986). 